\title{
A Generalized Local Time-Step Scheme for Efficient FVTD Simulations in Strongly Inhomogeneous Meshes
}

\author{
Christophe Fumeaux, Member, IEEE, Dirk Baumann, Student Member, IEEE, Pascal Leuchtmann, Member, IEEE, \\ and Rüdiger Vahldieck, Fellow, IEEE
}

\begin{abstract}
A new generalized local time-step scheme is introduced to improve the computational efficiency of the finite-volume time-domain (FVTD) method. The flexibility of unstructured FVTD meshes is fully exploited by avoiding the disadvantage of a single short time step in the entire mesh. The great potential of this scheme is fully revealed in the FVTD simulation of electromagnetic (EM) problems with both large and fine structures in close proximity. The scheme is based on an automatic partition of the computational domain in subdomains where local time steps of the type $2^{\ell-1} \Delta t(\ell=1,2,3, \ldots)$ can be applied without violating the stability condition. Interfaces between subdomains are reduced to a generic two-level system which requires a very limited number of time interpolations during the FVTD iteration, therefore resulting in a very simple and robust technique. The application of local time stepping to three-dimensional EM problems demonstrates a significant speed-up of the computation without compromising the accuracy of the results.
\end{abstract}

Index Terms-Computational electromagnetics, conformal meshing, dielectric antennas, finite-volume time-domain (FVTD) method, numerical analysis.

\section{INTRODUCTION}

$\mathbf{T}$ HE finite-volume time-domain (FVTD) method has been applied to the numerical solution of Maxwell's equations since the end of the 1980s [1], [2]. It has benefited from experience gathered previously in finite-volume techniques used in computational fluid dynamics. A great advantage of the FVTD method is its applicability in unstructured meshes. Therefore, FVTD constitutes a powerful alternative to the finite-difference time-domain (FDTD) method for electromagnetic (EM) problems where conformal meshing is advantageous, i.e., for structures that include curved or oblique surfaces. Another type of geometries that still constitute a challenge for time-domain methods are those in which fine structural details compared to the wavelength (i.e., with dimensions in the order of $\lambda / 100$ or even smaller) are in close proximity to larger structures. The discretization of such structures with three-dimensional (3-D) regular Cartesian grids results in prohibitively high computational costs. Subcell models and subgridding techniques have been developed for FDTD to handle these features, at

Manuscript received July 22, 2003; revised October 31, 2003. This work was supported by the Swiss Defense Procurement Agency (Armasuisse), Bern, Switzerland.

The authors are with the Laboratory for Electromagnetic Fields and Microwave Electronics, Swiss Federal Institute of Technology, 8092 Zürich, Switzerland (e-mail: fumeaux@ifh.ee.ethz.ch).

Digital Object Identifier 10.1109/TMTT.2004.823595 the cost of increased complexity, threat to stability, and loss of second-order accuracy. In contrast, using an unstructured mesh permits to adapt locally the cell dimensions to the size of the geometrical features, therefore avoiding a 3-D explosion of the number of cells. The transition between the regions with different cell densities is smooth in nature. However, the drawback that still remains is that the required time step for stability is determined by the smallest cell in the entire mesh. To overcome this problem for the FVTD method, we propose a generalized scheme of local time steps in an unstructured mesh. The proposed technique considers only discrete levels of time steps with lengths given by power-of-two multiples (i.e., 1, 2, 4, 8 ) of the smallest time step necessary for stability in the entire mesh. Through a fully automatic procedure, the computational domain is partitioned into subdomains where the different discrete time steps can be applied without compromising the stability of the computation. This processing of the mesh is based on geometrical properties of the cells and requires no extra considerations at the time of mesh generation. During the FVTD time-marching iteration, the update of the EM field is performed with different schedules in the different subdomains. The local time-step scheme described in this paper fits very naturally into the FVTD march-in-time algorithm, although boundaries between the different subdomains require special treatment with careful timing. The advantage over the subgridding techniques of FDTD (e.g., [3]-[6]) is that no spatial interpolations or time extrapolations of the field components are required.

Several examples will illustrate the performance of this scheme. In the first example, the propagation of a wave in a parallel-plate waveguide permits to characterize the numerical error at the boundary between subdomains. The second case considers wave propagation simulated in an extremely inhomogeneous mesh with nine nested local time-step subdomains. The third example is a practical application: the scheme is applied to a probe-fed dielectric resonator antenna (DRA) which includes material boundaries with a large contrast of dielectric permittivities. A substantial speed-up of computation is attained using local time steps without decreasing the accuracy of the simulation results.

\section{FVTD ALGORITHM}

Several variations of the FVTD algorithm exist for the numerical solution of EM problems. They are based on various 
approximations of an exact integral formulation of Maxwell's curl equations.

\section{A. Exact Formulation}

The FVTD formalism is based on Maxwell's curl equations written in their conservative form [7]. These equations are integrated over elementary volumes $V_{i}$ ("finite volumes") using the divergence theorem. This results in the volume-surface integral form

$$
\begin{aligned}
-\frac{\partial}{\partial t} \iiint_{V_{i}} \vec{B} d v & =\oiint_{\partial V_{i}} \vec{n} \times \vec{E} d a \\
\frac{\partial}{\partial t} \iiint_{V_{i}} \vec{D} d v & =\oiint_{\partial V_{i}} \vec{n} \times \vec{H} d a
\end{aligned}
$$

where $\partial V_{i}$ is the boundary enclosing the volume $V_{i}$ and $\vec{n}$ indicates the outward-pointing normal unit vector of the surface element $d a$. The integrands of the surface integrals on the right-hand side (RHS) of the equations are interpreted as "fluxes" through the boundary of the volume.

For numerical implementation, polyhedrons $V_{i}$ with $N_{i}$ planar faces are considered as elementary volumes. The volume integrals on the left-hand side (LHS) are represented through multiplying the volume $V_{i}$ by the values of $\vec{B}$ and $\vec{D}$ averaged over the volume $V_{i}$. On the RHS, the surface integrals are calculated by averaging the values of $\vec{E}$ and $\vec{H}$ over the area of each face, multiplying by the face area and summing up the contributions of all the polyhedron faces. Using $\langle\cdot\rangle$ to denote spatially averaged values, assuming constant permeability $\mu$ and permittivity $\varepsilon$ in $V_{i}$, and inserting the material equations for each cell, the discretized equation (1) is then written as

$$
\begin{aligned}
-\mu \frac{\partial}{\partial t}\langle\vec{H}\rangle_{V_{i}} & =\frac{1}{V_{i}} \sum_{k=1}^{N_{i}}\left(\vec{n}_{k} \times\langle\vec{E}\rangle_{F_{k}}\right) F_{k} \\
\varepsilon \frac{\partial}{\partial t}\langle\vec{E}\rangle_{V_{i}} & =\frac{1}{V_{i}} \sum_{k=1}^{N_{i}}\left(\vec{n}_{k} \times\langle\vec{H}\rangle_{F_{k}}\right) F_{k}
\end{aligned}
$$

where $F_{k}$ is the area of the $k$ th face and $\vec{n}_{k}$ is its outwardpointing unit vector. To simplify the notation, we have used $F_{k}$ instead of $F_{i, k}$ to denote the $k$ th face of the $i$ th volume, and similarly $\vec{n}_{k}$ instead of $\vec{n}_{i, k}$. It should be noted at this point that the coupled equations (2) that represent the foundation of the FVTD scheme are exact under our assumptions. They relate volumeaveraged EM-field components to surface-averaged EM-field components.

\section{B. Approximations}

Approximations of (2) are necessary to enable their processing in a computer. They introduce the numerical error in the FVTD scheme. In contrast to FDTD, which is based almost exclusively on the Yee scheme, a large variety of different FVTD schemes exist. The algorithm variations are basically distinguished by three characteristics: finite-volume discretization, flux approximation, and time-stepping scheme. These three characteristics are discussed in the following with indications of the options used in the algorithm presented here.

Discretization-Location of the Field Components: A 3-D polyhedral volume discretization is characterized by a set of nodes distributed in the computational domain. For example, in a tetrahedral mesh, each cell is defined by a subset of four nodes. Variant FVTD algorithms are characterized by different topological locations of the stored field components. In the cell-centered formulation, the volume-averaged field components are approximated by locating them in the barycenter $B C_{i}$ of the cell and the surface-averaged field components are positioned in the barycenter $B C_{k}$ of the considered face $k$, i.e.,

$$
\begin{aligned}
& \langle\vec{E}\rangle_{V_{i}} \approx \vec{E}_{B C_{i}} \quad\langle\vec{E}\rangle_{F_{k}} \approx \vec{E}_{B C_{k}} \\
& \langle\vec{H}\rangle_{V_{i}} \approx \vec{H}_{B C_{i}} \text { and }\langle\vec{H}\rangle_{F_{k}} \approx \vec{H}_{B C_{k}} .
\end{aligned}
$$

Another formulation of the FVTD algorithm (cell vertex) locates the volume-averaged field components in the nodes of the discretization and requires construction of a dual mesh. Some alternative formulations also consider staggered $\vec{E}$ - and $\vec{H}$-field locations [8].

The cell-centered formulation in a tetrahedral mesh, with collocated $\vec{E}$ - and $\vec{H}$-fields in the cell barycenters, is applied in this investigation. This has the significant advantage of treating boundaries naturally since they coincide with cell faces. Furthermore, the need to use a computationally costly dual mesh is avoided.

Flux Approximation: In most FVTD algorithms, a flux-splitting formulation is introduced using a plane-wave assumption: for each face of the cell, plane waves propagating in the direction of the normal vector are considered and permit a splitting of the flux into the following two contributions.

- The outgoing flux through a cell face is estimated on the basis of barycenter field values in the considered cell.

- The incoming flux through a cell face is estimated on the basis of barycenter field values in the neighbor cell sharing the face.

The flux-splitting formulation inserted in (2) yields the approximations

$$
\begin{aligned}
-\mu \frac{\partial}{\partial t}\langle\vec{H}\rangle_{V_{i}} & =\frac{1}{V_{i}} \sum_{k=1}^{N_{i}}\left(\vec{n}_{k} \times\langle\vec{E}\rangle_{F_{k}}^{+}-\vec{n}_{k} \times\langle\vec{E}\rangle_{F_{k}}^{-}\right) F_{k} \\
\varepsilon & \frac{\partial}{\partial t}\langle\vec{E}\rangle_{V_{i}}=\frac{1}{V_{i}} \sum_{k=1}^{N_{i}}\left(\vec{n}_{k} \times\langle\vec{H}\rangle_{F_{k}}^{+}-\vec{n}_{k} \times\langle\vec{H}\rangle_{F_{k}}^{-}\right) F_{k}
\end{aligned}
$$

where the superscripts + and - indicate contributions from plane waves propagating toward the outside and inside of $V_{i}$, respectively. The separation of flux is justified using characteristics theory and obtained in practice through splitting of the $6 \times 6$ flux matrix according to the sign of its eigenvalues. Explicit formulas describing this separation are given in [7].

Independently of the time-marching scheme used, an FVTD time step can basically be separated into the following two stages, as illustrated in Fig. 1.

1) The (tangential) field components on the faces of the cell are approximated from values at the barycenter of the considered cell and its direct neighbors. This step introduces a numerical error since it basically extracts surface-averaged values from volume-averaged values. 


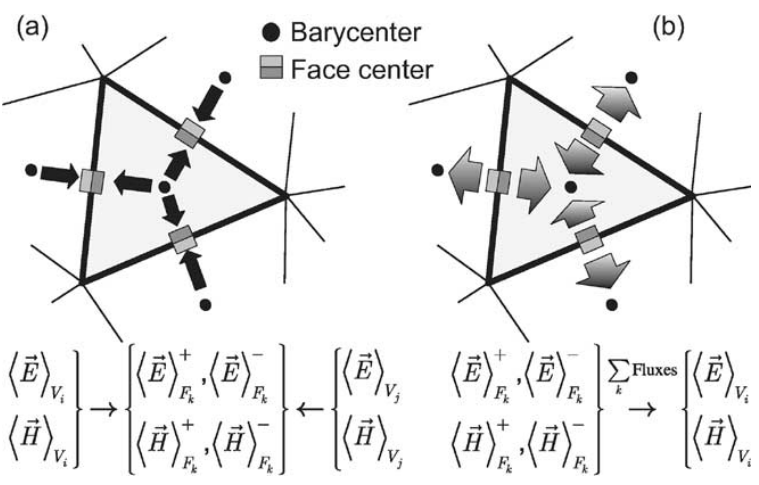

Fig. 1. Two-dimensional illustration of the two stages of one FVTD iteration step. (a) Barycenter values to face values. (b) Face values to barycenter values.

2) The barycenter values are updated using fluxes through the cell faces according to (4) and following the chosen time-marching scheme.

The flux-splitting formulation is applied in the investigation presented here. Together with the Monotonic Upwind Scheme for Conservation Laws (MUSCL) [7], this permits to achieve second-order accuracy in space by using estimated gradients in the barycenters of the cells.

As an alternative to flux splitting, we mention a scheme proposed in [9] which builds fluxes using averaged field components at the boundary between the cells.

Time Stepping: The fundamental FVTD equations (2) or (4) are discretized in space. The field components in theses equations are time-dependent vector functions [10]. The FVTD update equations are obtained introducing time steps denoted by an index $n$ and separated by a period $\Delta t$. After integrating (4) during one full time step, we can write

$$
\begin{aligned}
& \langle\vec{H}\rangle_{V_{i}}^{n+1}=\langle\vec{H}\rangle_{V_{i}}^{n}-\frac{\Delta t}{\mu V_{i}} \sum_{k=1}^{N_{i}}\left(\vec{n}_{k} \times \overline{\langle\vec{E}\rangle_{F_{k}}^{+}}-\vec{n}_{k} \times \overline{\langle\vec{E}\rangle_{F_{k}}^{-}}\right) F_{k} \\
& \langle\vec{E}\rangle_{V_{i}}^{n+1}=\langle\vec{E}\rangle_{V_{i}}^{n}+\frac{\Delta t}{\varepsilon V_{i}} \sum_{k=1}^{N_{i}}\left(\vec{n}_{k} \times \overline{\langle\vec{H}\rangle_{F_{k}}^{+}}-\vec{n}_{k} \times \overline{\langle\vec{H}\rangle_{F_{k}}^{-}}\right) F_{k}
\end{aligned}
$$

where the overbar indicates an average over one time step. These two equations permit to write "new" volume-averaged field components (LHS) as a function of "older" volume-averaged values (first term on the RHS) and surface-averaged field components averaged over one time step (second term on the LHS).

The various time-stepping schemes essentially approximate the time averages in (5). The simplest scheme approximates the time derivatives using finite differences (first-order accuracy in time). In the algorithm presented here, second-order accuracy in time is achieved using the Lax-Wendroff scheme that is composed of the two steps schematically depicted in Fig. 2. The predictor step is a half step that explicitly approximates the time-dependent function $u$ at time $n+1 / 2$ using values at time $n$. This half-step value is then used to perform a corrector full step, approximating the time-averaged values by values in the middle of the step.

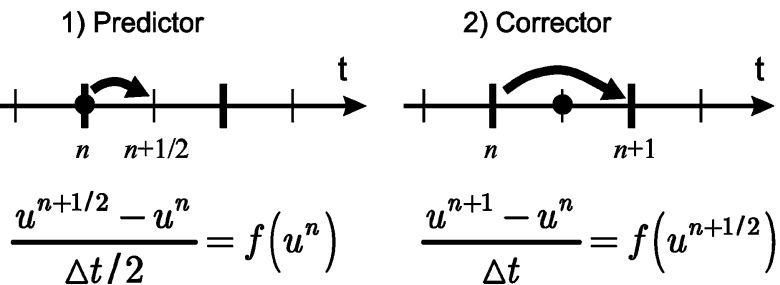

Fig. 2. Illustration of the two steps of the Lax-Wendroff time-stepping scheme for numerical solution of a differential equation of the type $\partial u / \partial t=f(u)$. The gray dots indicate when the function $f(u)$ is evaluated.

The stability criterion for the time-step length of the FVTD iteration is determined by the cell with the worst volume/surface ratio in the entire mesh according to [7]

$$
\Delta t \leq \frac{p}{v} \min _{i}\left(\frac{V_{i}}{\sum_{k=1}^{N_{i}} F_{k}}\right)
$$

where $v$ represents the wave velocity in the medium and $p$ is given by

$$
\begin{aligned}
\text { first-order scheme: } & p=1 \\
\text { Lax-Wendroff scheme: } & p=2 .
\end{aligned}
$$

This last equation reflects the fact that using the Lax-Wendroff second-order scheme permits us to double the time step for stability. Considering (6) in a tetrahedral mesh, it is obvious that the presence of small or flat tetrahedrons decreases the time step in the whole computational domain. Using local time steps with the scheme presented in this paper affects exclusively the time-stepping approximations.

\section{LOCAL TIME-STEP SCHEME}

The local time-step scheme presented in this section permits to exploit the full capability of meshes with inhomogeneous cell densities to model small structural details by avoiding the restriction of a single time step over the whole computational domain. The proposed technique is implemented in a generic fashion in the FVTD algorithm so that no external intervention is necessary.

\section{A. General Idea}

The basic principle of our scheme is to subdivide the computational domain into subdomains where local time steps with discrete values can be applied without violating the local stability condition. The interfaces between the different subdomains require special treatment since they involve interactions between adjacent cells that follow different update schedules. In order to realize a robust and fully automatic procedure, we propose a scheme that has the following main characteristics.

- Time Steps With Discrete Values $2^{\ell-1} \Delta t$ : Only predetermined levels of time steps are considered in the proposed algorithm. The chosen discrete values $\Delta t_{\ell}$ correspond to powers of two multiples of the fundamental time step $\Delta t$ 


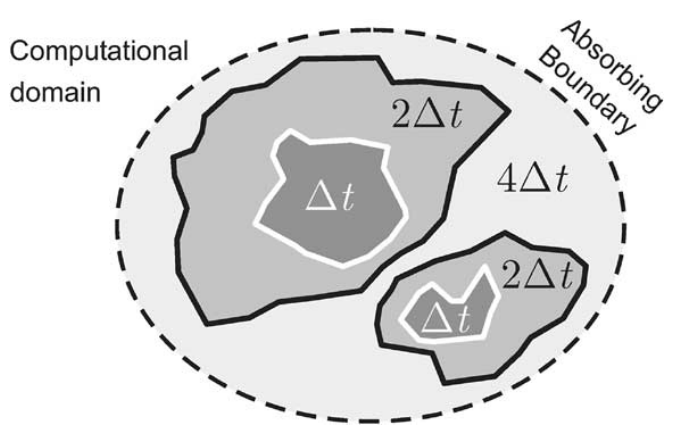

Fig. 3. Two-dimensional representation of the subdomain partition of a computational domain containing two small structures (not shown). The transition from regions with time step $\Delta t$ to regions with time step $4 \Delta t$ requires an intermediate zone with time step $2 \Delta t$. The boundaries (including adjacent cells) between contiguous domains are strictly separated so that they can be described as two-level systems.

determined through the maximum of criterion (6) on the entire mesh as follows:

$$
\Delta t_{\ell}=2^{\ell-1} \Delta t, \quad \ell=1,2,3, \ldots
$$

where $\ell$ denotes the time-step level. The time-marching FVTD algorithm uses the fundamental time step $\Delta t$, but the updating is only performed in selected domains at a certain time step.

- Local Stability: A requirement for the partition of the computational domain is that the stability condition (6) is satisfied locally. We define subdomains $\Omega_{\ell}$ where $\Delta t_{\ell}$ is applied. In $\Omega_{\ell}$, the local time step $\Delta t_{\ell}$ is shorter than required for stability in each cell.

- Two-Level Boundaries: A further requirement for the separation in subdomains is that the time-step jump between two adjacent subdomains is exactly a factor of two, as illustrated in Fig. 3. In addition, boundaries between different levels are strictly disjoint, i.e., a cell can only belong to one boundary.

- No Spatial Interpolations: The local time-step scheme presented here requires no spatial interpolation, which is in contrast to subgridding techniques developed for FDTD. In the unstructured FVTD mesh, the transition from small cells to large cells is continuous and introduces no topological change. The boundary between time-step subdomains is part of the original mesh.

\section{B. Preprocessing-Subpartition of the Computational Domain}

The partition of the computational domain in subsets $\Omega_{\ell}$ is performed fully automatically during a preprocessing step on the basis of local geometrical properties and requires no preconditioning while generating the mesh.

All tetrahedrons are temporarily assigned to the fundamental time step $\Delta t$. The subdomains $\Omega_{\ell}$ associated with the diverse levels $\ell$ of time steps are then defined through an iterative procedure: Tetrahedrons from level $\ell$ (starting at $\ell=1$ ) are "upgraded" to the level $\ell+1$ if they satisfy all three of the following conditions.

1) Local stability. The considered (single) tetrahedron is large enough so that the geometric stability condition (6) is satisfied at the double time step $2^{\ell} \Delta t$.

2) No double jumps. The same stability condition is also satisfied for all tetrahedrons in the "extended neigh-

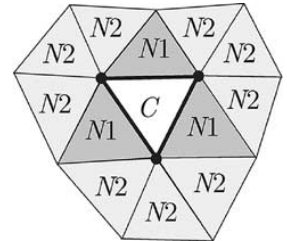

Fig. 4. Two-dimensional illustration of the neighborhood of a considered triangle $C$ (tetrahedron in 3-D) defined by its nodes (black dots). The direct neighborhood is formed by the cells $N 1$ that share a face with $C$. The extended neighborhood contains in addition the cells $N 2$ that share at least one node with $C$.

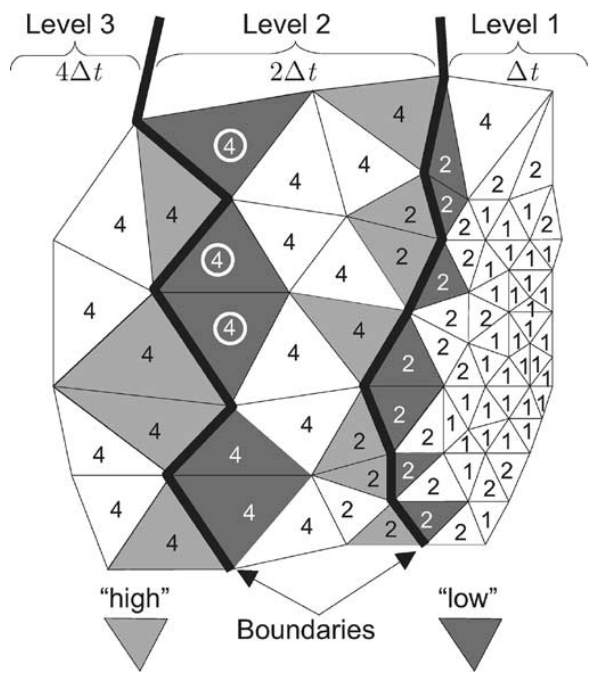

Fig. 5. Two-dimensional representation of the computational domain partition according to the local time-step level. The small digits in the cells indicate the multiple of the fundamental time step $\Delta t$ that could be used according to condition 1 only (stability condition for the single cell). The cells with white circles are not upgraded to level 3 because of condition 3 .

borhood" of the considered tetrahedron. The extended neighborhood contains all tetrahedrons sharing at least one node with the considered tetrahedron (typically 60-70 cells) as illustrated in two dimensions in Fig. 4.

3) Disjoint boundaries. None of the tetrahedrons in the "extended neighborhood" of the considered cell belongs to a boundary of the previous level, i.e., have a direct neighbor at level $\ell-1$.

It should be noted that all tetrahedrons are processed in parallel during one step of the level iteration. The iterative procedure continues until no tetrahedrons are left that satisfy the upgrade conditions to the next level.

The final step of the preprocessing extracts the boundary cells between the levels, since they need special treatment during iteration. This reduces the number of cases that need to be considered during the FVTD iteration (Fig. 5).

- Time-step level "bulk" tetrahedrons are surrounded by tetrahedrons that belong to the same time-step level (white cells in Fig. 5).

- Time-step level "low-boundary" tetrahedrons have one or several direct neighbors (cells $N 1$ in Fig. 4) with a $2 \times$ larger time step.

- Time-step level "high-boundary" tetrahedrons have one or several direct neighbors with a $2 \times$ smaller time step. 


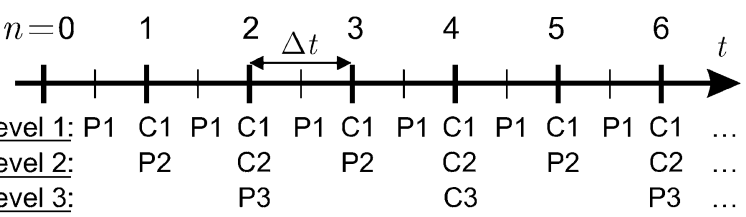

Fig. 6. Update schedule for a computational domain partitioned with three time-step levels. The $P$ 's indicate predictor steps and the $C$ 's indicate corrector steps.

Simultaneously satisfying the three conditions for the partition displaces the boundary toward larger cells, therefore providing some reserve for the stability criterion. Conditions 2) and 3) avoid the application of larger local time steps on isolated large tetrahedrons, which would not increase the efficiency since it involves more (computationally more expensive) "boundaries" than "bulk."

This general subdomain partition scheme can be applied without modification to other types of polyhedral meshes or for a stability condition different than (6). Even in a mesh with a fairly good homogeneity in cell sizes, it may permit an increase of efficiency, since the presence of a single cell of poor quality could result in a small fundamental time step.

\section{FVTD Iteration With Local Time Steps}

The FVTD time-marching algorithm is performed at the fundamental time step $\Delta t$. However, at the iteration step $n$, the field components are only updated in part of the subdomains. The local time-step treatment in the "bulk" and at the time-step boundaries is described separately in the following.

- Algorithm Inside the Subdomains (Time-Step Bulk): In the explicit FVTD update equation, a cell interacts at a time step $n$ only with its direct neighbors. This permits a straightforward application of the local time stepping in the bulk. When applying the Lax-Wendroff time-stepping scheme, the field components in all bulk cells of a domain $\Omega_{\ell}$ are updated according to the following rules:

$$
\begin{aligned}
& \text { predictor: if } \frac{n-2^{\ell-2}}{2^{\ell-1}} \text { is integer or } \ell=1 \\
& \text { corrector: if } \frac{n}{2^{\ell-1}} \text { is integer. }
\end{aligned}
$$

The update timetable in a three-level partition is given in Fig. 6 as an example. Depending on the time-marching scheme used, the rule can be slightly altered. For example, in the case of a first-order time-marching scheme, the update in the different subdomains would correspond to the "corrector" schedule given above.

- Treatment of the Boundaries: At the boundary between subdomains, a special treatment is necessary since numerical interactions occur between cells with different update schedules. The generic two-level update schedule in a Lax-Wendroff predictor-corrector scheme is illustrated in Fig. 7 at the boundary between levels 1 and 2. It basically consists of four distinct recurring stages (indicated by circles in Fig. 7).

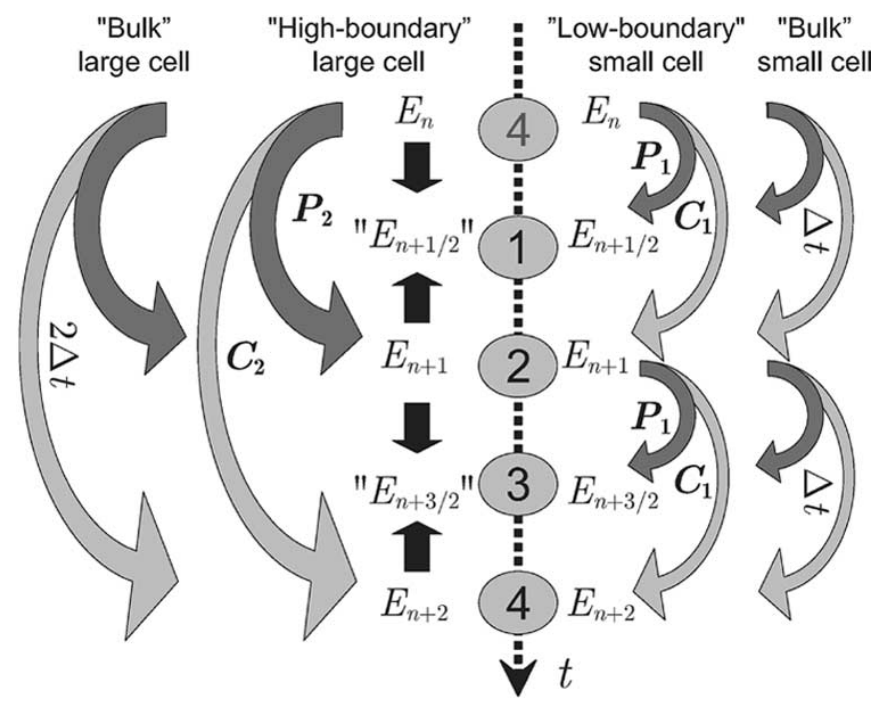

Fig. 7. Schematic representation of a two-level boundary system between subdomains with different time steps. An arbitrary field component is denoted by $E$ and the iteration step by $n$. The Lax-Wendroff predictor steps are denoted with dark grey arrows and the corrector steps with light gray arrows. The artificial half steps are indicated using black arrows.

Stage 1: The predictor step $P_{1}$ is applied in the smaller cells. All field components at the required time step $n$ are available in the computer memory for insertion in the explicit equations.

Stage 2: $\quad$ The predictor step $P_{2}$ is performed in the larger cells and the corrector step $C_{1}$ in the smaller cells. Since field values at half step $\left(E_{n+1 / 2}\right)$ are required for $C_{1}$ but are not available directly in the larger cells, an artificial half step needs to be created in the large cells after $P_{2}$

$$
\text { "E } E_{n+1 / 2} "=\frac{E_{n}+E_{n+1}}{2} .
$$

Stage 3: $\quad$ The predictor step $P_{1}$ is performed again. Note that, for the required field value $E_{n+1}$ in the large boundary cell (LHS in Fig. 7), the result of the predictor step $P_{2}$ in the larger cell is used.

Stage 4: The final stage consists of the corrector steps $C_{1}$ and $C_{2}$ in both large and small cells. As in stage 2, an artificial half step is required in the larger cells as follows:

$$
\text { "E } E_{n+3 / 2} "=\frac{E_{n+1}+E_{n+2}}{2} .
$$

The same procedure is applied at all time-step boundaries following the schedule required by the two levels involved. The computation of the gradient for second-order accuracy in space (the MUSCL scheme) has not been mentioned for the sake of brevity. It requires careful bookkeeping and precise timing in the frame of the local time-stepping scheme. All of the necessary data for the computation of the gradients can be obtained at the required time in the iteration using additional time approximations of the type (10) and (11) in the larger cells of the boundaries. 
The proposed scheme contains no spatial interpolation and very basic time interpolations. It is therefore strongly believed that, since each cell is updated at a schedule consistent for stability, a more severe stability criterion than the original one is not required. None of the examples computed in practice contradicted this assumption.

\section{Estimation of Simulation Speed-Up}

The use of local time steps permits us to achieve a significant speed-up of the computation in a strongly unstructured mesh since larger cells will be updated less often than smaller cells. The general dependence of the computation time on the level of the bulk cell is given by $1 / 2^{\ell-1}$. The cells at the boundaries between two time-step subdomains follow a similar dependence on their level $\ell$, however, with a CPU time overhead, since their treatment is computationally more demanding than bulk cells. Considering a problem that can be partitioned in $L$ levels of time steps, the speed-up factor $S$ associated with the use of local time steps can be estimated through the following formula:

$$
\frac{1}{S} \approx \sum_{\ell=1}^{L} \frac{N_{B}^{\ell}}{2^{\ell-1}}+\left(1+O_{L}\right) \sum_{\ell=1}^{L-1} \frac{N_{L}^{\ell}}{2^{\ell-1}}+\left(1+O_{H}\right) \sum_{\ell=2}^{L} \frac{N_{H}^{\ell}}{2^{\ell-1}}
$$

where $N_{B}^{\ell}$ represents the number of cells in the time-step bulk at level $\ell$, and $N_{L}^{\ell}$ and $N_{H}^{\ell}$ are the numbers in the low and high time-step boundaries, respectively, at level $\ell$. The overhead associated with the treatment of boundary cells is described by the additional constants $O_{L}$ and $O_{H}$ for the low- and high-boundary cells, respectively. These computation overheads can be determined empirically and typically yield $O_{L} \approx O_{H} \approx 1$.

Depending on the simulated problem, a limitation of the number of levels can prove to be beneficial for the efficiency. Such a behavior is observed when high-level subdomains have a large "boundary cells/bulk cells" ratio. The optimal number of levels can be automatically determined using (12) after the initial partition of the computational domain.

\section{NUMERICAL RESULTS}

The advantages of the local time-step scheme are demonstrated in the following by three simulation examples. For all of the examples chosen, an inhomogenous mesh is applied.

\section{A. Time-Step Transition in a Parallel-Plate Waveguide}

The numerical error introduced by a time-step transition has been investigated using propagation of a plane wave in a parallel-plate waveguide. The discretization is finer in one part of the cubic computational domain than in the other (Fig. 8). The typical ratio of linear dimensions between cells in both parts is two. The shaded zone in Fig. 8(a) represents the smooth transition between the two regions. The automatic local time-step subdomain partition procedure places a boundary in this zone between the level-1 region $(\Delta t)$ and the level-2 region $(2 \Delta t)$. A guided wave is excited either from small cells toward larger cells (wave 1 in Fig. 8) or vice versa (wave 2). The black dots in Fig. 8 mark the location of the cell barycenters where the field
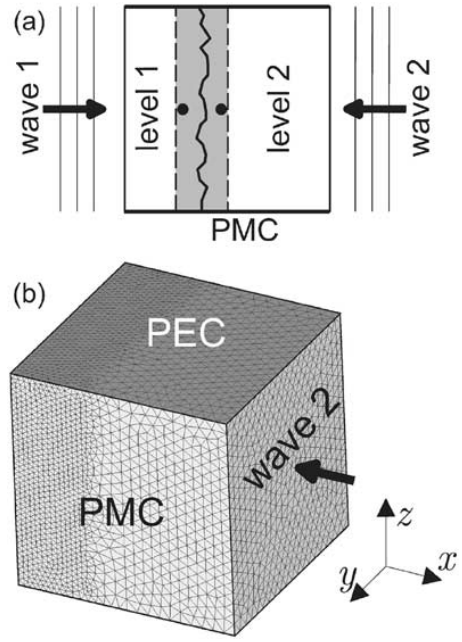

Fig. 8. (a) Top schematic representation of a parallel-plate waveguide with time-step boundary. Level-1 cells have linear dimensions approximately two times smaller than level-2 cells. Simulation of the wave incident from both sides has been performed. (b) 3-D representation of the cube outer surface mesh. The top and bottom boundaries are perfect electric conductors (PECs) and the left and right boundaries are perfect magnetic conductors (PMCs). The plane wave is polarized in the $z$ direction.
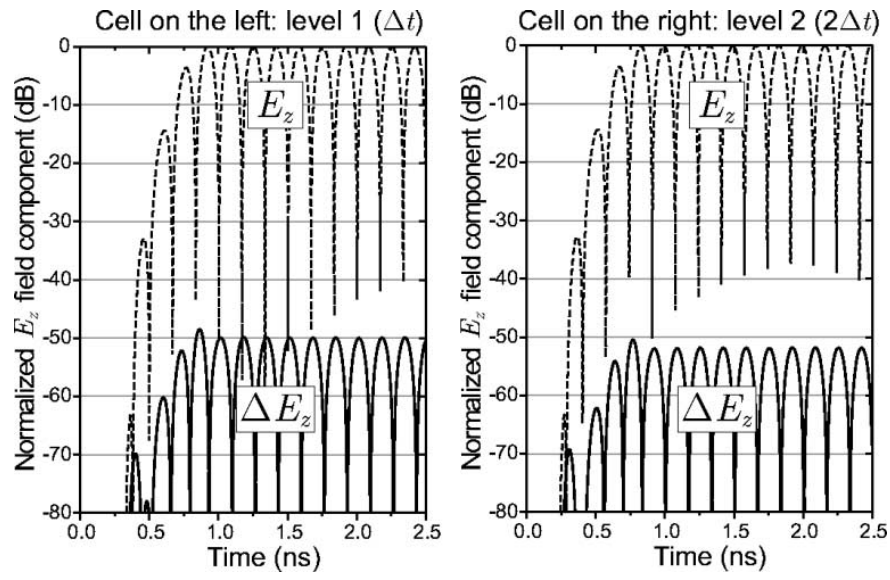

Fig. 9. Influence of the local time stepping on the field component $E_{z}$ at two points located on either side of the time-step boundary. The wave is traveling from cells with linear dimensions $\lambda / 10$ to smaller cells with dimensions $\lambda / 20$. $E_{z}$ represents the time-dependent field component simulated with local time steps and $\Delta E_{z}$ the deviation from the simulation performed without local time steps.

components are observed. The field components resulting from the simulation performed without local time stepping are considered a reference solution. The numerical error introduced by the application of local time steps is then the deviation from this reference.

Fig. 9 shows as an example the time dependence of the $E_{z}$ component of wave 2 simulated with local time steps. The magnitude of the error $\Delta E_{z}$ is on the order of $-50 \mathrm{~dB}$ in this particular case.

The dependence of the error on the discretization and on the direction of propagation is plotted in Fig. 10. The discretization is varied by changing the frequency of the guided wave. The error associated with the use of local time steps is decreasing with increasing fineness of discretization. Therefore, the first boundary in a system of nested subdomains will be the dominant 


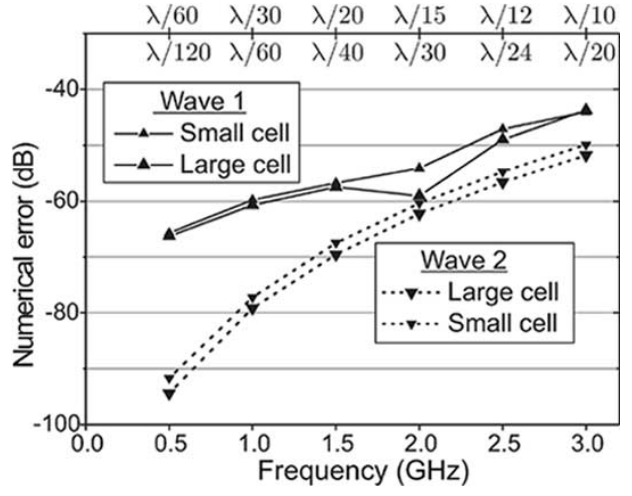

Fig. 10. Frequency dependence of the numerical error introduced by local time-stepping. Varying the frequency for a given mesh corresponds to changing the discretization: the upper horizontal scale gives the typical discretizations on both sides of the time-step transition.

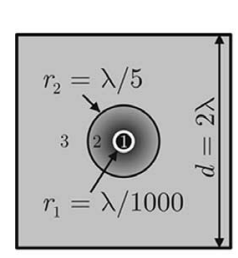

(a)

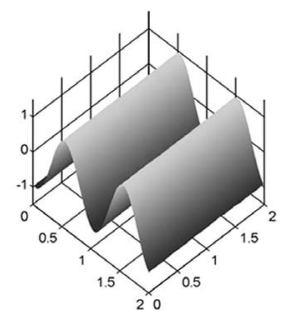

(b)
Fig. 11. (a) Schematic two-dimensional (2-D) representation (not scaled) of the 3-D computational domain with extremely unstructured mesh used for the simulation of plane-wave propagation. (b) Free-space 3-GHz plane wave simulated in the unstructured mesh with local time steps.

source of error. It is also observed that transitions toward larger cells (wave 1) show larger errors than transitions toward smaller cells (wave 2). The error is of the same order on both sides of the time-step transition (i.e., for large and small cells).

Judging from these results and from the observation of the dynamical behavior of the numerical error, it is believed that the main cause of error is not only due to pure reflections at the time-domain boundary. Other sources of errors contribute as well including phase errors inherent to the use of different time steps (similarly as in FDTD) and a phase shift caused by the different discretized time functions of the excitations. This can be understood considering (5): the discrete values obtained through simulations represent integrated values over a time step.

\section{B. Plane-Wave Propagation in an Extremely Inhomogeneous Mesh}

The second example considers the free-space propagation of a plane wave $(f=3 \mathrm{GHz})$ in a cubic computational domain. A large inhomogeneity of cell sizes is introduced artificially with the help of two spherical surfaces that define three domains in the cube (Fig. 11).

1) The first domain is defined by a tiny sphere with radius $r_{1}=\lambda / 1000$ placed in the center of the cube. The inner of this sphere is meshed with tetrahedrons with typical linear dimension close to $\lambda / 1600$.

2) The second domain is delimited by the first tiny sphere and by a larger sphere with radius $r_{2}=\lambda / 5$. This domain marks the transition between the very fine mesh inside

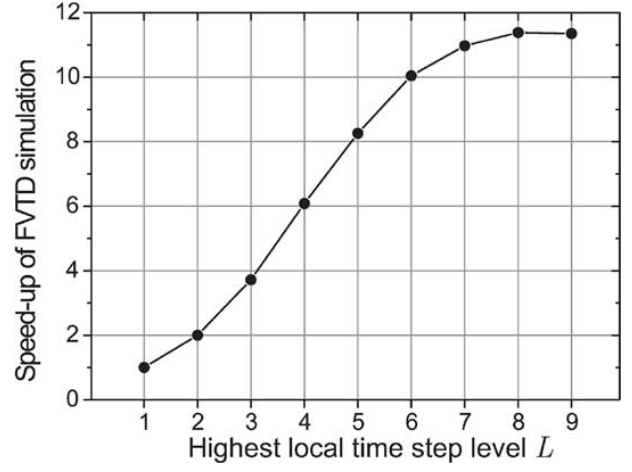

Fig. 12. Measured speed-up as a function of the highest time-step level used.

the smaller sphere $(\lambda / 1600)$ and the coarser mesh on the outside of the larger sphere $(\lambda / 10)$. Therefore, the mesh is extremely unstructure in this region

3) The third domain is delimited by the larger sphere and the cube that forms the outer boundary. The typical linear dimensions of the tetrahedrons are $\lambda / 10$.

The introduced spherical surfaces do not correspond to any material interface. However, their presence causes a significant inhomogeneity of cell sizes in the mesh: The typical linear dimensions are 160 times smaller in the center of the cube than close to the outer boundary, resulting in typical volume ratios of four million to one. A comparison of this highly distorted mesh with the same cube entirely meshed with $\lambda / 10$ fineness (i.e., in the absence of the small spheres) yields the following observations.

- The total number of cells increases only by a factor of 1.56 when the spheres are introduced, despite the occurrence of huge differences in cell volumes.

- The fundamental time step for stability $\Delta t$ is decreased by more than a factor of 100 , resulting in a dramatic slowing of the computation.

The application of local time steps in this extreme case permits to moderate this slowing of the computation. The plane-wave propagation has been first simulated in the strongly inhomogeneous mesh without using local time steps. In further simulations, local time steps were introduced gradually increasing to the highest level $L$ permitted. The measured speed-up of the computation as a function of $L$ is represented in Fig. 12 . Since the number of extremely small cells is very low, we observe that introducing a second level of time steps (that concerns more than $96 \%$ of the cells) already results in nearly halving the computation time. As the number of levels increases, we observe a saturation of the achievable speed-up, with a maximum value around 11.4. This saturation is caused by the fact that fewer and fewer cells satisfy all three upgrade conditions (Section III-B). The optimal efficiency in this example is obtained using eight levels $(128 \Delta t)$. The scheme would even permit us to use a ninth level $(256 \Delta t)$, but the overhead associated with the necessary additional time-step boundary would slightly decrease the overall efficiency of the computation, in accordance with (12). Fig. 13 shows the harmonic responses ( $E_{z}$-field component) in two different cells of the computational domain. The large cell in Fig. 13(a) has a local time step of $128 \Delta t$ whereas 


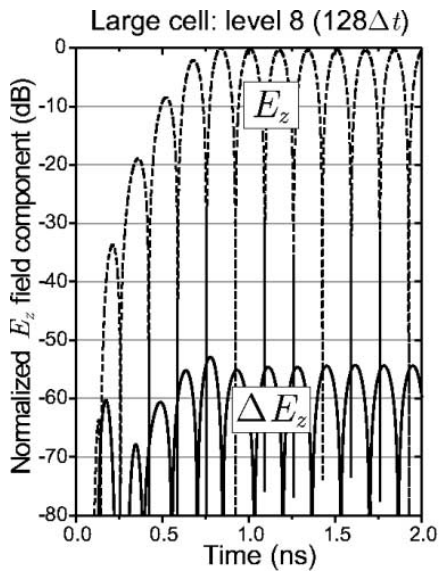

(a)

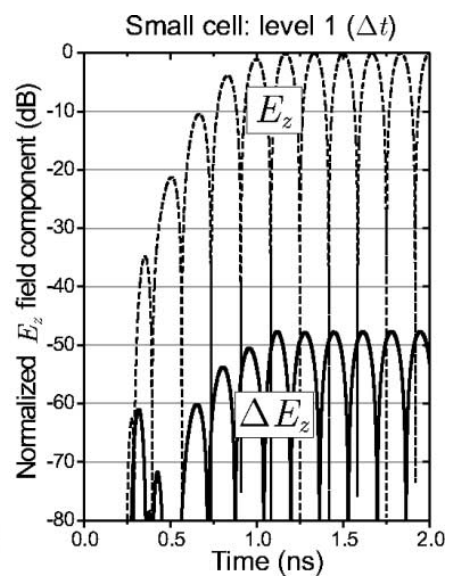

(b)
Fig. 13. Time-domain dependence of the plane-wave electric field at two different points. (a) At the barycenter of a large cell outside the spheres. (b) At the barycenter of a cell inside the tiny inner sphere. The field component $E_{z}$ simulated using local time steps and the difference $\Delta E_{z}$ to the reference simulation (without application of local time steps) are represented in the graphs.

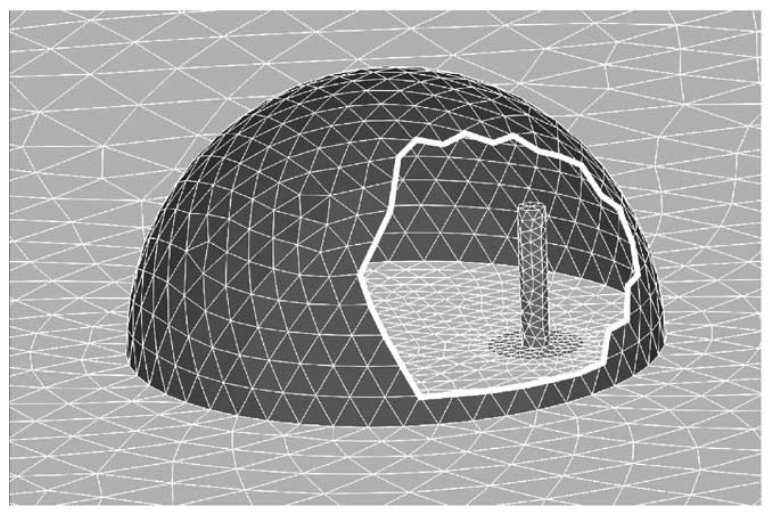

Fig. 14. Discretization of the hemispherical DRA. Only the triangulation of boundary surfaces is displayed. The hemisphere radius is $r=12.5 \mathrm{~mm}$ and the permittivity $\varepsilon_{r}=9.5$. Both the length of the probe and its displacement from the center of the hemisphere are equal to $6.5 \mathrm{~mm}$.

the tiny cell in Fig. 13(b) requires the fundamental time step $\Delta t$ since it is located in the center of the cube. The ratio of these two considered volumes is more than 3.5 million. Despite the presence of eight time-step boundaries between the two cells, the difference $\Delta E_{z}$ between the result of the simulations performed with and without local time stepping is very small. This demonstrates the accuracy and robustness of the proposed scheme even for large numbers of nested time-step subdomains.

\section{Hemispherical DRA}

The speed-up potential of the new local time-step scheme is also demonstrated for practical EM problems. The simulation of a probe-fed hemispherical DRA benefits from this technique since the structure includes a fine feed and a large dielectric contrast. The model used here is depicted in Fig. 14 and is based on the design of [11], with a radius $r=12.5 \mathrm{~mm}$ and a dielectric permittivity $\varepsilon_{r}=9.5$, resulting in a resonance frequency around 3.6 GHz.

The geometry of the hemispherical DRA is naturally modeled in an unstructured mesh with smooth transitions between
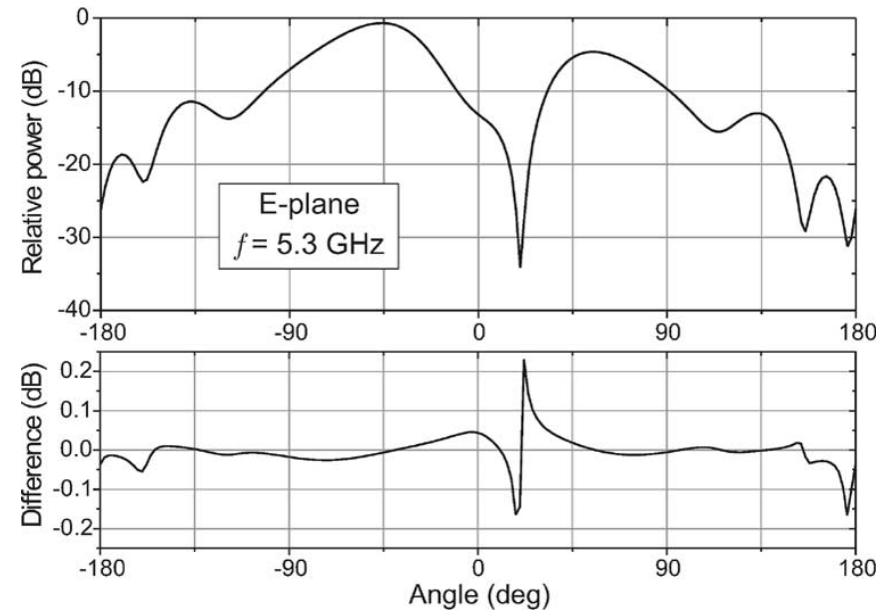

Fig. 15. Simulated radiation pattern of the DRA at $5.3 \mathrm{GHz}$ in the $E$-plane (containing the probe and the axis of the hemisphere). The lower graph shows the difference (in decibels) between the simulations performed with and without local time stepping.

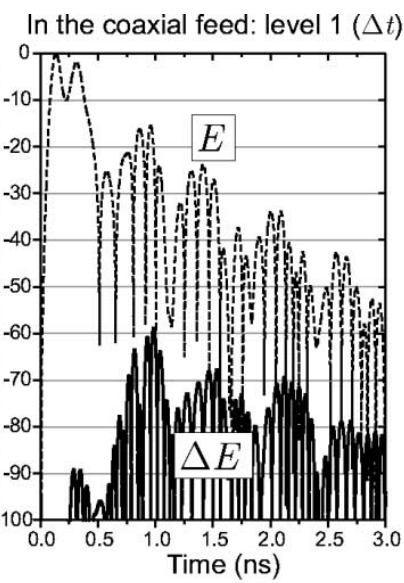

(a)

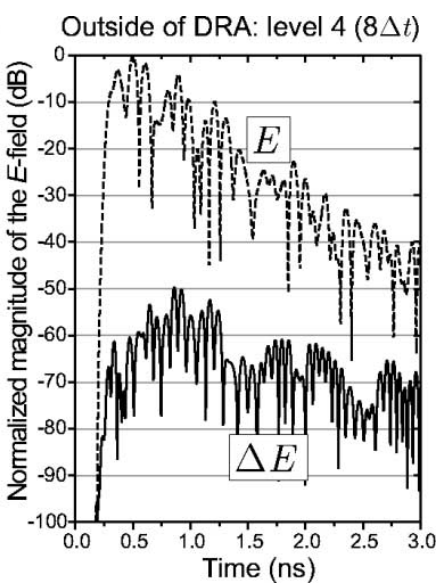

(b)
Fig. 16. Time-domain dependent magnitude of the electric field at the barycenter of two different cells of the computational domain. (a) The cell is located inside the coaxial feed. (b) The cell is outside of the DRA (free space). The graphs represent the magnitude of the $E$-field simulated using local time stepping and the difference $\Delta E$ with respect to the reference simulation without application of local time steps.

domains with smaller and larger cell densities. The FVTD simulation of this DRA from 3 to $6 \mathrm{GHz}$ has been described in [12], including sample plots of the return loss and radiation patterns. A speed-up of the computation by a factor of 3.7 is achieved applying local time steps for this simulation, demonstrating the advantage provided by the technique.

The influence of local time stepping on radiation patterns is insignificant. Fig. 15 shows an example of simulated pattern at $5.3 \mathrm{GHz}$ (a second resonance) together with the difference between simulations with and without local time stepping. The frequency was chosen because of the presence of a sharp minimum to illustrate the fact that discrepancies larger than $0.1 \mathrm{~dB}$ are only found near the direction of the radiation minima.

The influence of local time stepping is also almost imperceptible when considering the time-domain response to the modulated Gaussian pulse used for broad-band excitation. Fig. 16 shows the magnitude $E$ of the electric field observed at the 
TABLE I

SUMMARY OF RESULTS OBTAINED Using THE PRESENTED TIME-STEPPING SCHEME

\begin{tabular}{l|c|c|c|c}
\hline & $\begin{array}{c}\text { Plane wave in } \\
\text { extreme mesh }\end{array}$ & $\begin{array}{c}\text { Horn } \\
\mathbf{1 - 4} \mathbf{~ G H z} \\
\text { model [13] }\end{array}$ & $\begin{array}{c}\text { Horn } \\
\mathbf{4 - 1 8 ~ G H z} \\
\text { model [13] }\end{array}$ & DRA \\
\hline $\begin{array}{l}\text { Ratio of typical linear dimensions of } \\
\text { the cells (largest/smallest cells) }\end{array}$ & 160 & 12 & 5 & 18 \\
\hline $\begin{array}{l}\text { Maximum ratio of cell volumes } \\
V_{\text {max }} / V_{\text {min }}\end{array}$ & $27 \cdot 10^{6}$ & $8910^{3}$ & $3 \cdot 10^{3}$ & $119 \cdot 10^{3}$ \\
\hline $\begin{array}{l}\text { Optimal number of level } L \\
\text { (corresp. max. time step) }\end{array}$ & 8 & 5 & 3 & 4 \\
\hline $\begin{array}{l}\% \text { of bulk element in level 1 } \\
\text { (finest discretization) }\end{array}$ & $3.0 \%$ & $4.0 \%$ & $0.8 \%$ & $4.2 \%$ \\
\hline $\begin{array}{l}\% \text { of bulk element in level } L \\
\text { (coarsest discretization) }\end{array}$ & $60.0 \%$ & $55.9 \%$ & $91.2 \%$ & $49.6 \%$ \\
\hline $\begin{array}{l}\text { Total \% of time-step boundary cells } \\
\text { Memory increase }\end{array}$ & $9.7 \%$ & $13.7 \%$ & $6.1 \%$ & $3.6 \%$ \\
\hline Achieved simulation speed-up & $6.6 \%$ & $5.0 \%$ & $0.4 \%$ & $3.3 \%$ \\
\hline
\end{tabular}

barycenter of two cells: The cell in Fig. 16(a) is a small cell (level 1) located in the coaxial cable near the port of the antenna and the cell in Fig. 16(b) is a large cell (level 4) located in free space outside the DRA. The differences $\Delta E$ between the simulations performed with and without local time stepping are also plotted in the graphs. The small magnitude of these differences demonstrates the very low level of error introduced by local time stepping. This fact is also reflected in the return loss $\left(S_{11}\right)$ of the antenna, where differences between both simulations (with and without local time steps) are under $0.015 \mathrm{~dB}$ over the entire frequency range.

\section{Summary}

The effects of using the local time-stepping scheme is summarized in Table I for the examples presented in this section and for the double-ridged horn antenna presented in [13]. The most significant gain in computation efficiency is achieved for geometries with large inhomogeneities in cell sizes.

The computational load associated with the necessary partition of the computational domain can be summarized as follows. The partition procedure demands a small amount of CPU time during preprocessing and results in a small increase in required computer memory $(<10 \%)$ compared to the single-time-step algorithm. However, these drawbacks appear unimportant when weighted against the achieved speed-up of the FVTD simulation.

\section{CONCLUSION}

A new generalized local time-step scheme for the FVTD method has been presented. Significant savings of CPU time are possible without sacrificing the accuracy of the simulation when applied to EM problems where small and large structures are in close proximity. For slightly inhomogeneous meshes, the local time-step scheme is also beneficial since it relaxes the requirements on the mesh quality. The technique is based on a fully automatic partition of the computational domain in subsets where local time steps can be applied without deteri- orating stability. Only discrete local time-step values equal to power-of-two multiples of the fundamental time step $\Delta t$ are admitted. This permits to reduce all boundaries between subdomains to generic two-level boundaries with a time-step jump of a factor of two. The different subdomains follow different update schedules during the FVTD iteration, resulting in a strongly increased computation efficiency. Significant speed-up of FVTD simulations has been demonstrated for examples that involve tetrahedral meshes with large inhomogenities in cell sizes. The numerical error introduced by the scheme is dependent on the fineness of the mesh involved. In practical cases, even when a large number of time-step transitions are required, typical errors under $-50 \mathrm{~dB}$ have been demonstrated. The local time-stepping scheme has been applied in conjunction with FVTD using a particular time-marching algorithm (Lax-Wendroff) but the ideas presented could be adapted to other algorithms as well.

\section{REFERENCES}

[1] N. K. Madsen and R. W. Ziolkowski, "A three-dimensional modified finite volume technique for Maxwell's equations," Electromagnetics, vol. 10, pp. 147-161, 1990.

[2] V. Shankar, A. H. Mohammadian, and W. F. Hall, "A time-domain, finite-volume treatment for the Maxwell equations," Electromagnetics, vol. 10 , pp. $127-145,1990$

[3] M. Okoniewski, E. Okoniewska, and M. A. Stuchly, "Three-dimensional subgridding algorithm for FDTD," IEEE Trans. Antennas Propagat., vol. 45, pp. 422-429, Mar. 1997.

[4] D. Menditto, P. Tognolatti, and F. Bardati, "FDTD analysis in cylindrical coordinates of a TEM pyramidal horn antenna," in 12th Annu. Rev. Progress in Applied Computational Electromagnetics, 1996, pp. $1010-1022$.

[5] S. Xiao, R. Vahldieck, and H. Jin, "A fast two dimensional FDTD full-wave analyzer with adaptive mesh size," in IEEE MTT-S Int. Microwave Symp. Dig., Albuquerque, NM, 1992, pp. 783-786.

[6] S. Xiao and R. Vahldieck, "A fast FDTD analysis of guided wave structures using a continuously variable mesh with second order accuracy," JIETE Millimeter Wave Integrated Circuits, pp. 3-14, 1995.

[7] P. Bonnet, X. Ferrieres, B. L. Michielsen, P. Klotz, and J. L. Roumiguières, "Finite-volume time domain method," in Time Domain Electromagnetics, S. M. Rao, Ed. San Diego, CA: Academic, 1999, ch. 9 .

[8] M. Yang, Y. Chen, and R. Mittra, "Hybrid finite-difference/finitevolume time domain analysis for microwave integrated circuits with curved PEC surfaces using a nonuniform rectangular grid," IEEE Trans. Microwave Theory Tech., vol. 48, pp. 969-975, June 2000.

[9] S. Piperno, M. Remaki, and L. Fezoui, "A nondiffusive finite volume scheme for the three-dimensional Maxwell's equations on unstructured meshes," SIAM J. Numer. Anal., vol. 39, no. 6, pp. 2089-2108, 2001.

[10] P. Leuchtmann, C. Fumeaux, and D. Baumann, "Comparison of errors and stability in FDTD and FVTD," Adv. Radio Sci., vol. 1, pp. 87-92, 2003 (available under http://www.copernicus.org/URSI/ars/ars_published.htm).

[11] K. W. Leung, K. K. Tse, K. M. Luk, and E. K. N. Yung, "Cross-polarization characteristics of a probe-fed hemispherical dielectric resonator antenna," IEEE Trans. Antennas Propagat., vol. 47, pp. 1228-1230, July 1999.

[12] D. Baumann, C. Fumeaux, P. Leuchtmann, and R. Vahldieck, "Finitevolume time-domain (FVTD) method and its application to the analysis of hemispherical dielectric-resonator antennas," in IEEE MTT-S Int. Microwave Symp. Dig., 2003, pp. 985-988.

[13] C. Fumeaux, D. Baumann, P. Leuchtmann, and R. Vahldieck, "A generalized local time-step for the FVTD method for efficient simulation of microwave antennas," in 33rd Eur. Microwave Conf. Dig., 2003, pp. 467-470. 


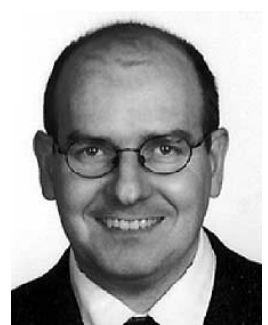

Christophe Fumeaux (M'03) received the Diploma and $\mathrm{Ph} . \mathrm{D}$. degrees in physics from the Swiss Federal Institute of Technology (ETH), Zürich, Switzerland, in 1992 and 1997, respectively. His doctoral dissertation concerned antenna-coupled infrared detectors.

From 1998 to 2000, he was a Post-Doctoral Researcher involved in infrared technology with the School of Optics, University of Central Florida, Orlando. In 2000, he joined the Swiss Federal Office of Metrology, Bern, Switzerland, as a Scientific Staff Member. Since 2001, he has been a Research Associate with the Laboratory for Electromagnetic Fields and Microwave Electronics (IFH), ETH, Zürich, Switzerland. His current main research interest concerns computational electromagnetics in the time domain for numerical analysis of microwave circuits and antennas.

Dr. Fumeaux was the recepient of the ETH Silver Medal of Excellence for his doctoral dissertation.

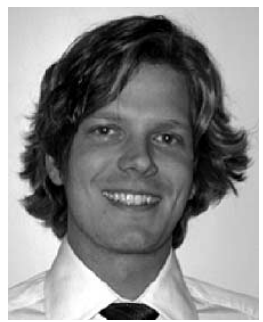

Dirk Baumann (S'01) received the Dipl. Ing degree in electrical engineering from the University of Karlsruhe, Karlsruhe, Germany, in 2001, and is currently working toward the Ph.D. degree in electrical engineering at the Swiss Federal Institute of Technology (ETH), Zürich, Switzerland

During the spring and fall of 2000, he held an internship with the Alaska SAR Facility (ASF), Fairbanks, Alaska, where he was involved with the calibration of the ASF's SAR processor. His research interests include numerical methods with emphasis on time-domain techniques and their application to general EM problems.

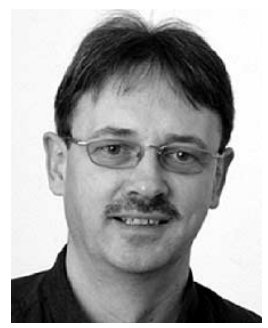

Pascal Leuchtmann (M'98) received the Diploma degree in electrical engineering and the Ph.D. degree from the Swiss Federal Institute of Technology (ETH), Zürich, Switzerland, in 1980 and 1987, respectively.

$\mathrm{He}$ is currently involved with EM compatibility and antennas research with the Laboratory for Electromagnetic Fields and Microwave Electronics, ETH.

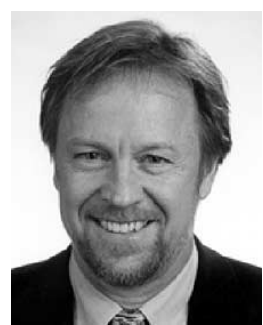

Ruediger Vahldieck (M'85-SM'86-F'99) received the Dipl.-Ing. and Dr.-Ing. degrees in electrical engineering from the University of Bremen, Bremen, Germany, in 1980 and 1983, respectively.

From 1984 to 1986 , he was a Post-Doctoral Fellow with the University of Ottawa, Ottawa, ON, Canada. In 1986, he joined the Department of Electrical and Computer Engineering, University of Victoria, Victoria, BC, Canada, where he became a Full Professor in 1991. During the fall of 1992 and the spring of 1993, he was a Visiting Scientist with the Ferdinand-Braun-Institute für Hochfrequenztechnik, Berlin, Germany. In 1997, he accepted an appointment as a Professor of EM-field theory with the Swiss Federal Institute of Technology (ETH), Zürich, Switzerland, and became Head of the Laboratory for Electromagnetic Fields and Microwave Electronics (IFH) in 2003. His research interests include computational electromagnetics in the general area of electromagnetic compability (EMC) and, in particular, for computer-aided design of microwave, millimeter-wave, and optoelectronic integrated circuits. Since 1981 he has authored or coauthored over 230 technical papers in books, journals, and conferences, mainly in the field of microwave computer-aided design.

Prof. Vahldieck is the past president of the IEEE 2000 International Zürich Seminar on Broadband Communications (IZS'2000). Since 2003, he has been president and general chairman of the International Zürich Symposium on Electromagnetic Compatibility. $\mathrm{He}$ is a member of the editorial board of the IEEE TRANSACTION ON MicrowaVe Theory and TeChNiques. From 2000 to 2003, he was an associate editor for the IEEE MICROWAVE AND WIRELESS COMPONENTS LETTERS, and in January 2004, he became the Editor-in-Chief. Since 1992, he has served on the Technical Program Committee (TPC) of the IEEE Microwave Theory and Techniques Society (IEEE MTT-S) International Microwave Symposium (IMS), the IEEE MTT-S Technical Committee on Microwave Field Theory, and in 1999, on the TPC of the European Microwave Conference. From 1998 to 2003, he was the chapter chairman of the IEEE Swiss Joint Chapter on Microwave Theory and Techniques, Antennas and Propagation, and EMC. He was the recipient of the J. K. Mitra Award of the Institution of Electronics and Telecommunication Engineers (IETE) (in 1996) for the best research paper in 1995 and was corecipient of the Outstanding Publication Award of the Institution of Electronic and Radio Engineers in 1983. 